\title{
Multi-period portfolio selection problem under uncertain environment with bankruptcy constraint
}

\author{
Bo Li*a, Yuanguo Zhu ${ }^{\mathrm{a}}$, Yufei Sun ${ }^{\mathrm{b}}$, Grace Aw ${ }^{\mathrm{b}}$, and Kok Lay Teo ${ }^{\mathrm{b}}$ \\ ${ }^{\mathrm{a}}$ School of Science, Nanjing University of Science and Technology, China \\ *Corresponding author: libnust@163.com \\ ${ }^{\mathrm{b}}$ Department of Mathematics and Statistics, Curtin University, Australia
}

\begin{abstract}
The complexity of financial markets leads to different types of indeterminate asset returns. For example, asset returns are considered as random variables, when the available data is enough. When the available data is too small or even no available data to estimate a probability distribution, we have to invite some domain experts to evaluate the belief degrees of asset returns. Then, asset returns can be described as uncertain variables. In this paper, we discuss a multi-period portfolio selection problem under uncertain environment, which maximizes the final wealth and minimizes the risk of investment. Unlike the common method to describe the multi-period portfolio selection problem as a bi-objective optimization model, we formulate this uncertain multi-period portfolio selection problem by a new method in three steps with two single objective optimization models. And, we consider the influence of transaction cost and bankruptcy of investor. Then, the proposed uncertain optimization models are transformed into the corresponding crisp optimization models and we use the genetic algorithm combined with penalty function method to solve them. Finally, a numerical example is given to show the effectiveness and practicability of proposed models and method.
\end{abstract}

Keywords: Multi-period portfolio selection; Uncertainty; Optimization; Investment strategy 


\section{Introduction}

In financial markets, the investor usually needs to choose an optimal investment strategy to allocate his/her wealth among different assets for achieving different investment goals, which is called the portfolio selection problem. The portfolio selection problem was pioneered by Markowitz [1] with the mean-variance portfolio selection model. Since then, the portfolio selection problem has been one of the most important optimization problems in financial markets. Many works have been done to study the single period portfolio selection problems, such as Sharpe [2], Pang [3], Best and Grauer [4], Simonelli [5], Sawik [6, 7, 8], Sun et al. [9].

However, in real world, most of investors are willing to invest long-term assets for gaining more return. That is, investors should adjust their investment strategies from time to time. Hence, the multi-period portfolio selection problems have attracted great attention of researchers. $\mathrm{Li}$ and $\mathrm{Ng}$ [10] considered an analytical optimal solution to the mean-variance formulation in multi-period portfolio selection problem. Zhu et al. [11] proposed a generalized mean-variance formulation from which an optimal investment policy can be generated to help investors not only achieve an optimal return, but also have a good risk control over bankruptcy. Calafiore [12] concerned with multi-period sequential decision problems for financial asset allocation and presented a multi-period portfolio optimization model with control policies. Costa and Araujo [13] dealt with a generalized multi-period mean-variance portfolio selection problem with market parameters subject to Markov random regime switchings. Wu and Li [14] investigated a multi-period mean-variance portfolio selection with regime switching and uncertain exit time. Wu and $\mathrm{Li}$ [15] studied a non-self-financing portfolio optimization problem under the framework of multi-period mean-variance with Markov regime switching and a stochastic cash flow. Sun et al. [16] developed a minimax model for a multi-period portfolio selection problem and provided a computationally simple analytical solution.

All the above literature assumes that the asset returns are random variables. The corresponding portfolio selection problems have been solved by probability theory. But, a premise of applying probability theory is that the available distribution is closed enough to the real frequency. However, we sometimes have no or not enough samples to estimate the distribution via statistics. Then, the fuzzy set theory offers an appropriate alternative. The fuzzy single period and multi-period portfolio selection problems have also been studied by many scholars. Vercher et al. [17] presented a fuzzy downside risk approach for managing portfolio selection problems in the framework of risk-return trade-off using interval-valued expectations. Li et al. [18] established a fuzzy mean-variance-skewness model and designed a genetic 
algorithm integrating fuzzy simulation. Sadjadi et al. [19] discussed a fuzzy multi-period portfolio optimization problem with different rates for borrowing and lending. Liu et al. [20] considered four fuzzy multi-period portfolio optimization models by using multiple criteria. Zhang et al. [21] presented a possibilistic mean-semivariance-entropy model for multi-period portfolio selection with fuzzy returns. Liu and Zhang [22] considered a multi-period fuzzy portfolio optimization problem with minimum transaction lots. Guo et al. [23] constructed a fuzzy multi-period portfolio selection problem under the assumption that risky assets have different investment horizons. Mehlawat [24] studied a fuzzy multi-objective multi-period portfolio selection problem considering multi-choice aspiration levels for each goal.

However, fuzzy theory suffers from criticism since a paradox will appear when fuzzy variable is used to describe the asset return (see Huang and Ying [25]). In order to better describe the subjective imprecise quantity, an uncertainty theory was founded by Liu [26] in 2007 and refined by Liu [27] in 2010 as another alternative tool to deal with human uncertainty. Then, many works have been done to develop the uncertainty theory, such as Liu [28], Yao and Li [29], Gao et al. [30], Yan and Zhu [31], Yao [32], Qin et al. [33]. Up to now, uncertainty theory has become a branch of axiomatic mathematics and contains many topics, such as uncertain programming, uncertain process, uncertain calculus, and uncertain differential equation. Meanwhile, many scholars have researched the portfolio selection problems based on uncertainty theory. For instance, Qin et al. [33] discussed an uncertain portfolio adjusting problem with risky assets. Zhu [34] introduced and dealt with an uncertain optimal control problem with application to a portfolio selection model. Ning et al. [35] proposed a new mean-TVaR model for portfolio selection, where the returns of securities are described as uncertain variables. Yao and Ji [36] proposed a decision-making method based on the expected utility criterion and used it to solve a portfolio selection problem. Ning et al. [37] considered a mean-variance portfolio selection problem with triangular entropy as a constraint. Furthermore, Huang and Qiao [38] employed uncertainty theory to help solve a multi-period portfolio selection problem, where the asset return rates are regarded as uncertain variables.

At present, there are few literature that focus on multi-period portfolio selection problem under the framework of uncertainty theory proposed by Liu [26] because of the complexity of transformation. Huang and Qiao [38] considered just a special case because they assumed that each asset return is a normal uncertain variable. In this paper, we will consider a multiperiod portfolio selection problem based on uncertainty theory by mean-variance model taking into account the transaction cost and bankruptcy of investor. Our paper contributes 
to the literature in the following way. Firstly, we employ the classical mean-variance model to consider the multi-period portfolio selection problem based on uncertainty theory. Secondly, the presented problem is formulated by a new method in three steps. Thirdly, our model holds for arbitrary uncertain variable with regular uncertainty distribution.

The rest of this paper is organized as follows. In Section 2, we review some concepts and theorems in uncertainty theory. The uncertain multi-period portfolio selection problem is modeled in three steps in Section 3. In Section 4, two proposed uncertain optimization models are converted into two crisp optimization models and a method is presented for solving them. A numerical example is given in Section 5. The last section gives a conclusion.

\section{Preliminary}

For formulating and discussing the multi-period portfolio selection problem under uncertain environment, many basic concepts and theorems in uncertainty theory should be used, such as uncertain variable, uncertainty distribution, and uncertain expected value. In order to facilitate understanding, in this section, we introduce all the needed concepts and theorems.

\section{Uncertain variable}

Let $\Gamma$ be a nonempty set, and $\mathcal{L}$ a $\sigma$-algebra over $\Gamma$. Each element $\Lambda \in \mathcal{L}$ is called an event. A set function $\mathcal{M}$ defined on the $\sigma$-algebra over $\mathcal{L}$ is called an uncertain measure if it satisfies the following axioms: (normality axiom) $\mathcal{M}\{\Gamma\}=1$ for the universal set $\Gamma$; (duality axiom) $\mathcal{M}\{\Lambda\}+\mathcal{M}\left\{\Lambda^{c}\right\}=1$ for any event $\Lambda$; (subadditivity axiom) $\mathcal{M}\left\{\bigcup_{i=1}^{\infty} \Lambda_{i}\right\} \leq \sum_{i=1}^{\infty} \mathcal{M}\left\{\Lambda_{i}\right\}$ for every countable sequence of events $\Lambda_{1}, \Lambda_{2}, \cdots$.

The triplet $(\Gamma, \mathcal{L}, \mathcal{M})$ is called an uncertainty space. A product uncertain measure $\mathcal{M}$ is defined by Liu [28] to produce an uncertain measure of compound event: (product axiom) Let $\left(\Gamma_{k}, \mathcal{L}_{k}, \mathcal{M}_{k}\right)$ be uncertainty spaces for $k=1,2, \cdots$. Then the product uncertain measure $\mathcal{M}$ is an uncertain measure satisfying

$$
\mathcal{M}\left\{\prod_{k=1}^{\infty} \Lambda_{k}\right\}=\bigwedge_{k=1}^{\infty} \mathcal{M}_{k}\left\{\Lambda_{k}\right\}
$$

where $\Lambda_{k}$ are arbitrarily chosen events from $\mathcal{L}_{k}$ for $k=1,2, \cdots$, respectively.

An uncertain variable is defined by Liu [26], i.e., an uncertain variable is a function $\xi$ from an uncertainty space $(\Gamma, \mathcal{L}, \mathcal{M})$ to the set of real numbers such that the set $\{\xi \in B\}=$ $\{\gamma \in \Gamma \mid \xi(\gamma) \in B\}$ is an event for any Borel set of real numbers. The uncertain variables 
$\xi_{1}, \xi_{2}, \cdots, \xi_{m}$ are said to be independent if

$$
\mathcal{M}\left\{\bigcap_{i=1}^{m}\left\{\xi_{i} \in B_{i}\right\}\right\}=\min _{1 \leq i \leq m} \mathcal{M}\left\{\xi_{i} \in B_{i}\right\}
$$

for any Borel sets $B_{1}, B_{2}, \cdots, B_{m}$ of real numbers.

The uncertainty distribution $\Phi(x)$ of an uncertain variable $\xi$ is defined by Liu [26] as

$$
\Phi(x)=\mathcal{M}\{\xi \leq x\}
$$

Then, Liu [27] presented the concept of regular uncertainty distribution, i.e., an uncertainty distribution $\Phi(x)$ is said to be regular if it is a continuous and strictly increasing function with respect to $x$ at which $0<\Phi(x)<1$, and

$$
\lim _{x \rightarrow-\infty} \Phi(x)=0, \quad \lim _{x \rightarrow+\infty} \Phi(x)=1 .
$$

When uncertainty distribution $\Phi(x)$ of an uncertain variable $\xi$ is regular, the inverse function $\Phi^{-1}(\alpha)$ is called the inverse uncertainty distribution of $\xi$.

An uncertain variable $\xi$ is called linear if it has a linear uncertainty distribution

$$
\Phi(x)=\left\{\begin{array}{cl}
0, & \text { if } x \leq a \\
(x-a) /(b-a), & \text { if } a \leq x \leq b \\
1, & \text { if } x \geq b
\end{array}\right.
$$

denoted by $\xi \sim \mathcal{L}(a, b)$ where $a$ and $b$ are real numbers with $a<b$. The inverse uncertainty distribution of linear uncertain variable $\mathcal{L}(a, b)$ is

$$
\Phi^{-1}(\alpha)=(1-\alpha) a+\alpha b, \quad(0<\alpha<1) .
$$

Theorem 1. (Liu [27]) Let $\xi_{1}, \xi_{2}, \cdots, \xi_{n}$ be independent uncertain variables with regular uncertainty distributions $\Phi_{1}, \Phi_{2}, \cdots, \Phi_{n}$, respectively. If the function $f\left(x_{1}, x_{2}, \cdots, x_{n}\right)$ is strictly increasing with respect to $x_{1}, x_{2}, \cdots, x_{m}$ and strictly decreasing with respect to $x_{m+1}, x_{m+2}, \cdots, x_{n}$, then $\xi=f\left(\xi_{1}, \xi_{2}, \cdots, \xi_{n}\right)$ is an uncertain variable with inverse uncertainty distribution

$$
\Psi^{-1}(\alpha)=f\left(\Phi_{1}^{-1}(\alpha), \cdots, \Phi_{m}^{-1}(\alpha), \Phi_{m+1}^{-1}(1-\alpha), \cdots, \Phi_{n}^{-1}(1-\alpha)\right)
$$




\section{Expected value and variance}

For describing the average value of an uncertain variable $\xi$, Liu [26] defined the concept of expected value as

$$
E[\xi]=\int_{0}^{+\infty} \mathcal{M}\{\xi \geq x\} \mathrm{d} x-\int_{-\infty}^{0} \mathcal{M}\{\xi \leq x\} \mathrm{d} x
$$

provided that at least one of the two integrals is finite.

Theorem 2. (Liu [26]) Let $\xi$ be an uncertain variable. Then for any given numbers a $>0$ and $p>0$, we have

$$
\mathcal{M}\{|\xi|>a\} \leq \frac{E\left[|\xi|^{p}\right]}{a^{p}}
$$

Theorem 3. (Liu [27]) Let $\xi$ be an uncertain variable with regular uncertainty distribution $\Phi$. Then

$$
E[\xi]=\int_{0}^{1} \Phi^{-1}(\alpha) \mathrm{d} \alpha .
$$

The definition of variance of an uncertain variable is also given by Liu [26] in 2007. Let $\xi$ be an uncertain variable with finite expected value $e$. Then the variance of $\xi$ is

$$
V[\xi]=E\left[(\xi-e)^{2}\right]
$$

Because of the subadditivity of uncertain measure, the variance of an uncertain variable cannot be deduced simply by its uncertainty distribution (see Liu [27]). Hence, Liu [27] defined

$$
V[\xi]=\int_{0}^{\infty}(1-\Phi(E[\xi]+\sqrt{x})+\Phi(E[\xi]-\sqrt{x})) \mathrm{d} x
$$

as an acceptable stipulation. Based on this stipulation, Yao [32] deduced the following theorem.

Theorem 4. (Yao [32]) Let $\xi$ be an uncertain variable with regular uncertainty distribution $\Phi$ and finite expected value $e$. Then

$$
V[\xi]=\int_{0}^{1}\left(\Phi^{-1}(\alpha)-e\right)^{2} \mathrm{~d} \alpha .
$$

\section{Problem formulation}

Here, we consider a multi-period portfolio problem with $N$ risky assets and $T$ investment periods in financial markets, where the return rate $r_{i, t}(i=1,2, \cdots, N, t=1,2, \cdots, T)$ is 
modeled as an uncertain variable with regular uncertainty distribution $\Phi_{i, t}$. Assume that the uncertain return rates $r_{i_{1}, t_{1}}$ and $r_{i_{2}, t_{2}}$ are independent for any different $i_{1}$ and $i_{2}\left(i_{1}, i_{2}=\right.$ $1,2, \cdots, N)$ or $t_{1}$ and $t_{2}\left(t_{1}, t_{2}=1,2, \cdots, T\right)$. The investor allocates his/her initial wealth $W_{0}$ among $N$ risky assets at beginning of period 1 and the wealth can be reallocated at every beginning of the following $T-1$ consecutive time periods. The purpose of investor is to maximize the final wealth and minimize the risk of investment. Assume that the whole investment process is self-financing, i.e., there is no exogenous infusion or withdrawal of money after the initial time. In order to formulate this mathematical model, we use the following notations:

$r_{i, t}$ : the uncertain return rate of risky asset $i$ at period $t$,

$x_{i, t}$ : the investment proportion of risky asset $i$ at the beginning of period $t$, $\boldsymbol{x}_{t}$ : the portfolio at the beginning of period $t$, where $\boldsymbol{x}_{t}=\left(x_{1, t}, x_{2, t}, \cdots, x_{N, t}\right)$,

$R_{t}$ : the return rate of portfolio $\boldsymbol{x}_{t}$ at period $t$, $c_{i, t}$ : the unit transaction cost of risky asset $i$ at period $t$, $W_{t}$ : the wealth at end of period $t$, where $i=1,2, \cdots, N$ and $t=1,2, \cdots, T$.

The return rate of portfolio $\boldsymbol{x}_{t}=\left(x_{1, t}, x_{2, t}, \cdots, x_{N, t}\right)$ at period $t$ can be expressed as

$$
R_{t}=\sum_{i=1}^{N} r_{i, t} x_{i, t}, \quad t=1,2, \cdots, T
$$

And, the transaction cost at the beginning of period $t$ is

$$
C_{t}=\sum_{i=1}^{N} c_{i, t}\left|x_{i, t}-x_{i, t-1}\right|, \quad t=1,2, \cdots, T .
$$

Here, we assume that $x_{i, 0}=0, i=1,2, \cdots, N$, which means that the investor held no assets before investment. Hence, the total wealth at the end of period $t$ is

$$
\begin{aligned}
W_{t} & =W_{t-1}\left(1+R_{t}-C_{t}\right) \\
& =W_{t-1}\left(1+\sum_{i=1}^{N} r_{i, t} x_{i, t}-\sum_{i=1}^{N} c_{i, t}\left|x_{i, t}-x_{i, t-1}\right|\right), \quad t=1,2, \cdots, T
\end{aligned}
$$

Obviously, the final wealth can be written as

$$
W_{T}=W_{0} \prod_{t=1}^{T}\left(1+\sum_{i=1}^{N} r_{i, t} x_{i, t}-\sum_{i=1}^{N} c_{i, t}\left|x_{i, t}-x_{i, t-1}\right|\right) .
$$


As it is well known, in stock markets, the circuit-breaker mechanism can be used to stabilize the stock markets and control the transaction risk. For controlling the risk of investment, we introduce the concept of bankruptcy, which means the total wealth of an investor falls below a preset "disaster" level in any period $t$. We stipulate that when the bankruptcy happens, the investor will terminate further investment behavior. Let $b_{t}, t=$ $1,2, \cdots, T$, be the preset bankruptcy threshold at period $t$. Then, the bankruptcy event at period $t$ can be denoted as $\Lambda_{t}=\left\{W_{t} \leq b_{t}, W_{j}>b_{j}, j=1,2, \cdots, t-1\right\}, t=1,2, \cdots, T$. The belief degree of uncertain event $\Lambda_{t}$ can be represented by

$$
\mathcal{M}\left\{\Lambda_{t}\right\}=\mathcal{M}\left\{W_{t} \leq b_{t}, W_{j}>b_{j}, j=1,2, \cdots, t-1\right\}, \quad t=1,2, \cdots, T .
$$

Proposition 1. Assume that $E\left[W_{t}\right]>b_{t}$ and $\frac{E\left[\left(W_{t}-E\left[W_{t}\right]\right)^{2}\right]}{\left(E\left[W_{t}\right]-b_{t}\right)^{2}} \leq \theta_{t}$. Then, it holds that $\mathcal{M}\left\{\Lambda_{t}\right\} \leq \theta_{t}$.

Proof Let $\xi=W_{t}-E\left[W_{t}\right], a=E\left[W_{t}\right]-b_{t}$ and $p=2$ in Theorem 2. Then, we have

$$
\mathcal{M}\left\{\left|W_{t}-E\left[W_{t}\right]\right| \geq E\left[W_{t}\right]-b_{t}\right\} \leq \frac{E\left[\left(W_{t}-E\left[W_{t}\right]\right)^{2}\right]}{\left(E\left[W_{t}\right]-b_{t}\right)^{2}} \leq \theta_{t} .
$$

Therefore,

$$
\begin{aligned}
\mathcal{M}\left\{\Lambda_{t}\right\} & =\mathcal{M}\left\{W_{t} \leq b_{t}, W_{j}>b_{j}, j=1,2, \cdots, t-1\right\} \\
& \leq \mathcal{M}\left\{W_{t} \leq b_{t}\right\} \\
& \leq \mathcal{M}\left\{\left|W_{t}-E\left[W_{t}\right]\right| \geq E\left[W_{t}\right]-b_{t}\right\} \\
& \leq \frac{E\left[\left(W_{t}-E\left[W_{t}\right]\right)^{2}\right]}{\left(E\left[W_{t}\right]-b_{t}\right)^{2}} \leq \theta_{t} .
\end{aligned}
$$

The proof is completed.

According to Proposition 1, the risk of bankruptcy at each period can be restricted by following equations

$$
E\left[W_{t}\right]>b_{t}, \quad t=1,2, \cdots, T,
$$

and

$$
E\left[\left(W_{t}-E\left[W_{t}\right]\right)^{2}\right] \leq \theta_{t}\left(E\left[W_{t}\right]-b_{t}\right)^{2}, \quad t=1,2, \cdots, T,
$$

where $0<\theta_{t}<1$, determined by the risk tolerance level of investor.

Generally, the risk and return of investment are positively related. In financial markets, it is almost impossible to achieve the maximum final wealth and minimum risk at the same time. Hence, the common method is to formulate the multi-period selection problem as a bi-objective optimization model, such as Li and Ng [10], Liu et al. [20], Zhang and Liu [43]. 
Then, taking a linear combination of the final wealth and risk functions with a weighting parameter, the bi-objective optimization model can be converted into a single objective optimization model. The weighting parameter can be chosen according to different risk preference. With the chosen weighting parameter, the two objective functions are combined to form a single objective which is to be optimized. For a conservative investor, he/she will first find the maximum final wealth that can be achieved without taking into consideration of the risk of he/she investment. Then the investor will seek to minimize the risk of his/her investment subject to the final wealth being not less than a specified acceptable final wealth for which the percentage of reduction from the maximum final wealth is determined by the investor. Clearly, the approach based on the choice of weighting parameter is inadequate.

Here, we formulate this uncertain multi-period portfolio selection problem in three steps. The final wealth and risk of investment are measured by uncertain expected value and variance, respectively.

Step 1: Without considering the risk of investment, we seek to find the maximum final wealth subject to the constraint equations (21) and (22) being satisfied. This problem is referred to as problem $\mathbf{P}_{1}$ :

$$
\begin{array}{ll}
\max & E\left[W_{T}\right]=E\left[W_{0} \prod_{t=1}^{T}\left(1+\sum_{i=1}^{N}\left(r_{i, t} x_{i, t}-c_{i, t}\left|x_{i, t}-x_{i, t-1}\right|\right)\right)\right] \\
\text { s.t. } & W_{t}=W_{t-1}\left(1+\sum_{i=1}^{N}\left(r_{i, t} x_{i, t}-c_{i, t}\left|x_{i, t}-x_{i, t-1}\right|\right)\right), \quad t=1,2, \cdots, T, \\
& E\left[\left(W_{t}-E\left[W_{t}\right]\right)^{2}\right] \leq \theta(t)\left(E\left[W_{t}\right]-b_{t}\right)^{2}, \quad t=1,2, \cdots, T \\
& E\left[W_{t}\right]>b_{t}, \quad t=1,2, \cdots, T \\
& \sum_{i=1}^{N} x_{i, t}=1, \quad t=1,2, \cdots, T \\
& 0 \leq x_{i, t} \leq q_{i, t}, \quad i=1,2, \cdots, N, t=1,2, \cdots, T,
\end{array}
$$

where $q_{i, t}$ is a preset upper bound of investment proportion $x_{i, t}$. In this step, we can obtain the maximum final wealth $E\left[W_{T}\right]^{*}$.

Step 2: We set a specified percentage of the maximum final wealth $E\left[W_{T}\right]^{*}$ as an acceptable final wealth, where the percentage of reduction from the maximum final wealth is determined by the investor. Then, we seek to minimize the risk of investment such that the constraints (21) and (22) are satisfied and the final wealth is not less that the acceptable 
final wealth. This problem is named as problem $\mathbf{P}_{2}$ :

$$
\begin{array}{ll}
\min & V\left[W_{T}\right]=V\left[W_{0} \prod_{t=1}^{T}\left(1+\sum_{i=1}^{N}\left(r_{i, t} x_{i, t}-c_{i, t}\left|x_{i, t}-x_{i, t-1}\right|\right)\right)\right] \\
\text { s.t. } & W_{t}=W_{t-1}\left(1+\sum_{i=1}^{N}\left(r_{i, t} x_{i, t}-c_{i, t}\left|x_{i, t}-x_{i, t-1}\right|\right)\right), \quad t=1,2, \cdots, T, \\
& E\left[\left(W_{t}-E\left[W_{t}\right]\right)^{2}\right] \leq \theta(t)\left(E\left[W_{t}\right]-b_{t}\right)^{2}, \quad t=1,2, \cdots, T, \\
& E\left[W_{t}\right]>b_{t}, \quad t=1,2, \cdots, T, \\
& \sum_{i=1}^{N} x_{i, t}=1, \quad t=1,2, \cdots, T, \\
& 0 \leq x_{i, t} \leq q_{i, t}, \quad i=1,2, \cdots, N, t=1,2, \cdots, T, \\
& E\left[W_{T}\right] \geq \rho E\left[W_{T}\right]^{*},
\end{array}
$$

where $0<\rho<1$ is to be determined by the investor. In this step, we can obtain the optimal investment strategies $\boldsymbol{x}_{1}^{*}, \boldsymbol{x}_{2}^{*}, \cdots, \boldsymbol{x}_{T}^{*}$ and the minimum risk of investment $V\left[W_{T}\right]^{*}$.

Step 3: Substituting $\boldsymbol{x}_{1}^{*}, \boldsymbol{x}_{2}^{*}, \cdots, \boldsymbol{x}_{T}^{*}$ into equation (23), the final wealth $E\left[W_{T}\right]$ can be calculated.

Remark 1. For a bold investor, he/she will seek to maximize the final wealth while bearing an acceptable risk of investment. The corresponding multi-period selection problem can be formulated conversely, i.e., the first step is to minimize the risk of investment without taking into consideration of the final wealth. In second step, an acceptable level for the risk of investment will be set, where the percentage of the increase of the risk of investment is determined by the investor. Then, the investor will seek to maximize his/her investment while the risk of his/her investment being maintained to be less than or equal to the acceptable level. In third step, the actual risk of investment can be calculated by using the optimal investment strategy obtained in Step 2. Clearly, this problem can also be solved by a similar method proposed in this paper.

\section{Deterministic transformation}

In this section, we transform the uncertain optimization problems $\mathbf{P}_{1}$ and $\mathbf{P}_{2}$ into the corresponding crisp optimization problems such that they can be solved more easily. 
Denote

$$
\begin{aligned}
& \varepsilon_{t}=\max \left\{c_{1, t}, c_{2, t}, \cdots, c_{N, t}\right\}, \quad t=1,2, \cdots, T, \\
& u_{t}=1+\sum_{i=1}^{N}\left(r_{i, t} x_{i, t}-c_{i, t}\left|x_{i, t}-x_{i, t-1}\right|\right), \quad t=1,2, \cdots, T .
\end{aligned}
$$

Theorem 5. Assume that $\mathcal{M}\left\{r_{i, t}>2 \varepsilon_{t}-1\right\}=1$ for $i=1,2, \cdots, N, t=1,2, \cdots, T$. Then, it holds that $\mathcal{M}\left\{u_{t}>0\right\}=1$.

Proof Obviously, we have $\sum_{i=1}^{N}\left|x_{i, t}-x_{i, t-1}\right| \leq 2$. Since $\varepsilon_{t}=\max \left\{c_{1, t}, c_{2, t}, \cdots, c_{N, t}\right\}$, it follows that

$$
\sum_{i=1}^{N} c_{i, t}\left|x_{i, t}-x_{i, t-1}\right| \leq \sum_{i=1}^{N} \varepsilon_{t}\left|x_{i, t}-x_{i, t-1}\right| \leq 2 \varepsilon_{t} .
$$

From $\mathcal{M}\left\{r_{i, t}>2 \varepsilon_{t}-1\right\}=1$, we obtain

$$
\mathcal{M}\left\{\sum_{i=1}^{N} r_{i, t} x_{i, t}>\sum_{i=1}^{N}\left(2 \varepsilon_{t}-1\right) x_{i, t}\right\}=1, \quad \text { i.e., } \quad \mathcal{M}\left\{\sum_{i=1}^{N} r_{i, t} x_{i, t}>2 \varepsilon_{t}-1\right\}=1 .
$$

Hence, we have $\mathcal{M}\left\{u_{t}>0\right\}=1, t=1,2, \cdots, T$. This completes the proof.

In the following discussion, for reducing the excessive risk of investment, we assume that for $i=1,2, \cdots, N, t=1,2, \cdots, T, \mathcal{M}\left\{r_{i, t}>2 \varepsilon_{t}-1\right\}=1$. According to Theorem 5, we know that $u_{j}$ is a positive uncertain variable with regular uncertainty distribution. It follows from Theorem 1 that $\prod_{j=1}^{t} u_{j}$ is also an uncertain variable with regular uncertainty distribution. The inverse uncertainty distribution of uncertain variable $\prod_{j=1}^{t} u_{j}$ is

$$
\Psi_{t}^{-1}(\alpha)=\prod_{j=1}^{t}\left(1+\sum_{i=1}^{N}\left[\Phi_{i, j}^{-1}(\alpha) x_{i, j}-c_{i, j}\left|x_{i, j}-x_{i, j-1}\right|\right]\right),
$$

where $\Phi_{i, j}^{-1}(\alpha)$ is the inverse uncertainty distribution of uncertain return rate $r_{i, t}$. From equations (10) and (13), for $t=1,2, \cdots, T$, we have

$$
\begin{aligned}
& E\left[W_{t}\right]=W_{0} \int_{0}^{1} \Psi_{t}^{-1}(\alpha) \mathrm{d} \alpha, \quad E\left[W_{T}\right]=W_{0} \int_{0}^{1} \Psi_{T}^{-1}(\alpha) \mathrm{d} \alpha \\
& E\left[\left(W_{t}-E\left[W_{t}\right]\right)^{2}\right]=V\left[W_{t}\right]=W_{0}^{2} \int_{0}^{1}\left(\Psi_{t}^{-1}(\alpha)-\int_{0}^{1} \Psi_{t}^{-1}(\alpha) \mathrm{d} \alpha\right)^{2} \mathrm{~d} \alpha \\
& V\left[W_{T}\right]=W_{0}^{2} \int_{0}^{1}\left(\Psi_{T}^{-1}(\alpha)-\int_{0}^{1} \Psi_{T}^{-1}(\alpha) \mathrm{d} \alpha\right)^{2} \mathrm{~d} \alpha .
\end{aligned}
$$


The uncertain optimization problems $\mathbf{P}_{1}$ and $\mathbf{P}_{2}$ are equivalent to following two models:

$$
\begin{array}{ll}
\max & E\left[W_{T}\right]=W_{0} \int_{0}^{1} \Psi_{T}^{-1}(\alpha) \mathrm{d} \alpha \\
\text { s.t. } & W_{0}^{2} \int_{0}^{1}\left(\Psi_{t}^{-1}(\alpha)-\int_{0}^{1} \Psi_{t}^{-1}(\alpha) \mathrm{d} \alpha\right)^{2} \mathrm{~d} \alpha \leq \theta(t)\left(W_{0} \int_{0}^{1} \Psi_{t}^{-1}(\alpha) \mathrm{d} \alpha-b_{t}\right)^{2}, \\
& \\
& W_{0} \int_{0}^{1} \Psi_{t}^{-1}(\alpha) \mathrm{d} \alpha>b_{t}, \quad t=1,2, \cdots, T, \\
& \sum_{i=1}^{N} x_{i, t}=1, \quad t=1,2, \cdots, T, \\
& 0 \leq x_{i, t} \leq q_{i, t}, \quad i=1,2, \cdots, N, t=1,2, \cdots, T,
\end{array}
$$

and

$$
\begin{array}{ll}
\min & V\left[W_{T}\right]=W_{0}^{2} \int_{0}^{1}\left(\Psi_{T}^{-1}(\alpha)-\int_{0}^{1} \Psi_{T}^{-1}(\alpha) \mathrm{d} \alpha\right)^{2} \mathrm{~d} \alpha \\
\text { s.t. } & W_{0}^{2} \int_{0}^{1}\left(\Psi_{t}^{-1}(\alpha)-\int_{0}^{1} \Psi_{t}^{-1}(\alpha) \mathrm{d} \alpha\right)^{2} \mathrm{~d} \alpha \leq \theta(t)\left(W_{0} \int_{0}^{1} \Psi_{t}^{-1}(\alpha) \mathrm{d} \alpha-b_{t}\right)^{2}, \\
& \\
& W_{0} \int_{0}^{1} \Psi_{t}^{-1}(\alpha) \mathrm{d} \alpha>b_{t}, \quad t=1,2, \cdots, T, \\
& \sum_{i=1}^{N} x_{i, t}=1, \quad t=1,2, \cdots, T, \\
& 0 \leq x_{i, t} \leq q_{i, t}, \quad i=1,2, \cdots, N, t=1,2, \cdots, T \\
& W_{0} \int_{0}^{1} \Psi_{T}^{-1}(\alpha) \mathrm{d} \alpha \geq \rho E\left[W_{T}\right]^{*},
\end{array}
$$

respectively.

\section{Solution method}

Obviously, the models (30) and (32) are essentially two nonlinear programming models. Constraint (31) can be written as:

$$
0 \leq x_{N, t}=1-\sum_{i=1}^{N-1} x_{i, t} \leq q_{N, t}, \quad t=1,2, \cdots, T .
$$


Then, the models (30) and (32) can be simplified as the following classical nonlinear constraint programming model:

$$
\begin{array}{ll}
\min & f(\boldsymbol{x}) \\
& g_{r}(\boldsymbol{x}) \leq 0, \quad r=1,2, \cdots, n, \\
& \boldsymbol{x} \in \Re^{m} .
\end{array}
$$

Using penalty function method, we construct an auxiliary function as:

$$
F(\boldsymbol{x}, \lambda)=f(\boldsymbol{x})+\lambda P(\boldsymbol{x})
$$

where the weighting parameter $\lambda$ is to be chosen sufficiently large, and

$$
P(\boldsymbol{x})=\sum_{r=1}^{n}\left[\max \left\{0, g_{r}(\boldsymbol{x})\right\}\right]^{2} .
$$

Hence, the nonlinear constraint programming model (34) can be transformed into an approximate nonlinear unconstraint programming model:

$$
\begin{array}{ll}
\min & F(\boldsymbol{x}) \\
& \boldsymbol{x} \in \Re^{m} .
\end{array}
$$

The genetic algorithm was proposed by Holland [39] in 1975. Since then, it has always been recognized as an effective and practical method for solving nonlinear programming problems. Nowadays, the genetic algorithm has still been used by many researchers for solving portfolio selection optimization problems, such as Li et al. [18], Huang [40], Yan et al. [41], Wang et al. [42], Zhang and Liu [43].

By the above penalty function method, the models (30) and (32) can be transformed into two approximate nonlinear unconstraint programming models. Then, we use genetic algorithm to solve two transformed models, where the procedures of the genetic algorithm are summarized as follows:

Step 1: Initialize randomly generated pop_size feasible chromosomes;

Step 2: Calculate the evaluation function values for all chromosomes;

Step 3: Perform the selection process by tournament selection method;

Step 4: Update the chromosomes by crossover and mutation operations;

Step 5: Repeat Steps 2-4 for a given number of generations;

Step 6: Report the best chromosome as the optimal solution. 


\section{Numerical simulation}

In this section, a numerical example is given to illustrate the practicality of the proposed models and the effectiveness of the solution method. Assume that an investor selects eight stocks in stock market market for which he/she wishes to invest for four quarters (one year), that is, $N=8$ and $T=4$. Then, the corresponding mathematical model can be formulated as in Section 3.

Here, the return rate $r_{i, t}(i=1,2, \cdots, 8, t=1,2,3,4)$ is modeled as an uncertain linear variable with $r_{i, t} \sim \mathcal{L}\left(a_{i, t}, b_{i, t}\right)$, as shown in Table 1 . The initial wealth $W_{0}=1$ and the upper bound of investment proportion are all set as 0.4 , i.e., $q_{i, t}=0.4, i=1,2, \cdots, 8, t=1,2,3,4$. The unit transaction costs are $c_{1, t}=0.0010, c_{2, t}=0.0015, c_{3, t}=0.0018, c_{4, t}=0.0020$, $c_{5, t}=0.0025, c_{6, t}=0.0032, c_{7, t}=0.0040$ and $c_{8, t}=0.0045$, for $t=1,2,3,4$. We assume that the bankruptcy will happen when the wealth of investor is zero, i.e., $b_{t}=0, t=1,2,3,4$, and the belief degree of bankruptcy is no higher than 0.2 , i.e., $\theta_{t}=0.2, t=1,2,3,4$. Also, the acceptable final wealth is set as $70 \%$ of maximum final wealth, i.e., $\rho=0.7$.

Since the genetic algorithm is a stochastic search method, the optimal solutions can be different with different choice of parameters being chosen in the algorithm. Here, we choose the best solution among the results of 20 times simulation. By using genetic algorithm (Matlab platform) combined with penalty function method (parameters are given in Table 2), the maximum final wealth, optimal investment strategy and minimum risk of investment can be obtained. From Table 3, we can find that, without considering the risk of investment, the maximum final wealth for investor is 1.2690. After obtaining the maximum final wealth, we can solve the optimal investment strategy and minimum risk of investment with the constraint of final wealth, where the acceptable final wealth is set as $70 \%$ of maximum final wealth, i.e., 0.8883. The obtained optimal investment strategies are

$$
\begin{aligned}
& \boldsymbol{x}_{1}^{*}=(0.3675,0.0145,0.1899,0.0976,0.1816,0.0809,0.0590,0.0090), \\
& \boldsymbol{x}_{2}^{*}=(0.3753,0.0423,0.1552,0.1552,0.1098,0.1115,0.0152,0.0355), \\
& \boldsymbol{x}_{3}^{*}=(0.3946,0.1802,0.0119,0.0771,0.0805,0.0133,0.1437,0.0987), \\
& \boldsymbol{x}_{4}^{*}=(0.3904,0.1172,0.1011,0.0736,0.0645,0.0405,0.0395,0.1732),
\end{aligned}
$$

as shown in Table 4. When the investor allocates his/her wealth according to the optimal investment strategy in Table 4, the minimum risk of investment is 0.1320 , and the final wealth is 1.1570 , which is more than the specified acceptable final wealth of 0.8883 . In addition, we calculate the maximum final wealth, minimum risk of investment and optimal investment proportions for five different weighting $\lambda$, as shown in Table 5 . Obviously, the 
Table 1: The parameters of uncertain return rates of eight assets at each period

\begin{tabular}{cccccccc}
\hline Period & Asset & $a_{i, t}$ & $b_{i, t}$ & & Asset & $a_{i, t}$ & $b_{i, t}$ \\
\hline $\mathrm{t}=1$ & Asset 1 & -0.06 & 0.07 & $\mathrm{t}=2$ & Asset 1 & -0.03 & 0.05 \\
& Asset 2 & -0.05 & 0.10 & & Asset 2 & -0.07 & 0.14 \\
& Asset 3 & -0.07 & 0.14 & & Asset 3 & -0.09 & 0.20 \\
& Asset 4 & -0.09 & 0.15 & & Asset 4 & -0.13 & 0.26 \\
& Asset 5 & -0.13 & 0.17 & & Asset 5 & -0.15 & 0.25 \\
& Asset 6 & -0.19 & 0.25 & & Asset 6 & -0.27 & 0.30 \\
& Asset 7 & -0.25 & 0.33 & & Asset 7 & -0.30 & 0.32 \\
& Asset 8 & -0.27 & 0.35 & & Asset 8 & -0.32 & 0.35 \\
\hline \multirow{2}{\mathrm{t}=3}{ 3 } & Asset 1 & -0.04 & 0.06 & $\mathrm{t}=4$ & Asset 1 & -0.08 & 0.10 \\
& Asset 2 & -0.03 & 0.08 & & Asset 2 & -0.11 & 0.20 \\
& Asset 3 & -0.05 & 0.09 & & Asset 3 & -0.15 & 0.23 \\
& Asset 4 & -0.05 & 0.10 & & Asset 4 & -0.16 & 0.28 \\
& Asset 5 & -0.10 & 0.20 & & Asset 5 & -0.20 & 0.37 \\
& Asset 6 & -0.14 & 0.20 & & Asset 6 & -0.25 & 0.40 \\
& Asset 7 & -0.20 & 0.26 & & Asset 7 & -0.30 & 0.41 \\
& Asset 8 & -0.24 & 0.34 & & Asset 8 & -0.32 & 0.38 \\
\hline
\end{tabular}

Table 2: The parameters of genetic algorithm and penalty function method

\begin{tabular}{ccccc}
\hline Population size & Iteration & Crossover probability & Mutation probability & $\lambda$ \\
\hline 50 & 1000 & 0.80 & 0.10 & $10^{9}$
\end{tabular}

Table 3: The optimal investment proportions and final wealth

\begin{tabular}{ccccccccc}
\hline Period & Asset 1 & Asset 2 & Asset 3 & Asset 4 & Asset 5 & Asset 6 & Asset 7 & Asset 8 \\
\hline $\mathrm{t}=1$ & 0.0013 & 0.0978 & 0.2129 & 0.0394 & 0.1295 & 0.1221 & 0.1668 & 0.2302 \\
$\mathrm{t}=2$ & 0.0295 & 0.2940 & 0.1273 & 0.1623 & 0.0221 & 0.1226 & 0.1050 & 0.1372 \\
$\mathrm{t}=3$ & 0.0027 & 0.0285 & 0.0836 & 0.2626 & 0.3618 & 0.1014 & 0.1055 & 0.0539 \\
$\mathrm{t}=4$ & 0.0024 & 0.0256 & 0.1008 & 0.0591 & 0.3213 & 0.1613 & 0.2912 & 0.0383 \\
\hline $\mathrm{FW}$ & & \multicolumn{7}{c}{1.2690} \\
\hline
\end{tabular}

bigger the weighting $\lambda$ is, the bigger the final wealth will become. Because different values of $E\left[W_{T}\right]^{*}$ lead to different constraints in problem $\mathbf{P}_{2}$, there is no change regulation for the risk of investment.

As shown in Figs. 1 and 2, the objective values of expected final wealth and risk of investment are converged to the optimal values after running 480 and 500 iterations, respectively, indicating that the genetic algorithm combined with penalty function method is an effective approach to solve the two proposed models. 
Table 4: The optimal investment proportions and final risk

\begin{tabular}{|c|c|c|c|c|c|c|c|c|}
\hline Period & Asset 1 & Asset 2 & Asset 3 & Asset 4 & Asset 5 & Asset 6 & Asset 7 & Asset 8 \\
\hline$t=1$ & 0.3675 & 0.0145 & 0.1899 & 0.0976 & 0.1816 & 0.0809 & 0.0590 & 0.0090 \\
\hline $\mathrm{t}=2$ & 0.3753 & 0.0423 & 0.1552 & 0.1552 & 0.1098 & 0.1115 & 0.0152 & 0.0355 \\
\hline $\mathrm{t}=3$ & 0.3946 & 0.1802 & 0.0119 & 0.0771 & 0.0805 & 0.0133 & 0.1437 & 0.0987 \\
\hline $\mathrm{t}=4$ & 0.3904 & 0.1172 & 0.1011 & 0.0736 & 0.0645 & 0.0405 & 0.0395 & 0.1732 \\
\hline FR & & & & 0.1320 & & & & \\
\hline
\end{tabular}

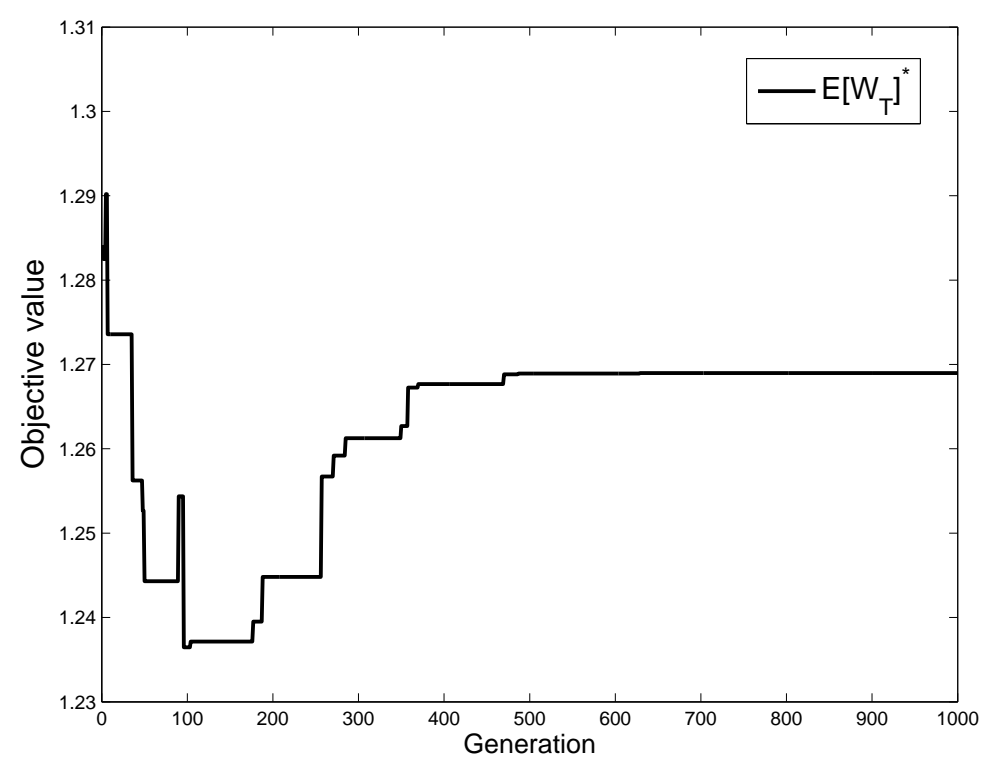

Figure 1: The convergence of genetic algorithm for optimal expected value.

\section{Conclusion}

In this paper, we investigated an uncertain multi-period portfolio selection problem in three steps with the influence of transaction cost and bankruptcy of investor being considered, where the final wealth and risk of investment are measured by uncertain expected value and variance, respectively. In view of the risk tolerance of investors, we formulated the uncertain multi-period portfolio selection problem in three steps. Then, two proposed uncertain optimization models were transformed into two crisp optimization models. And, we used a genetic algorithm combined with penalty function method to solve them. A numerical example with eight new stocks and four investment periods was given to demonstrate the effectiveness of the proposed models and method. The results showed that the proposed models and method are practical for multi-period portfolio selection problem when the available data is too small or even no available data to estimate the probability distributions of return 


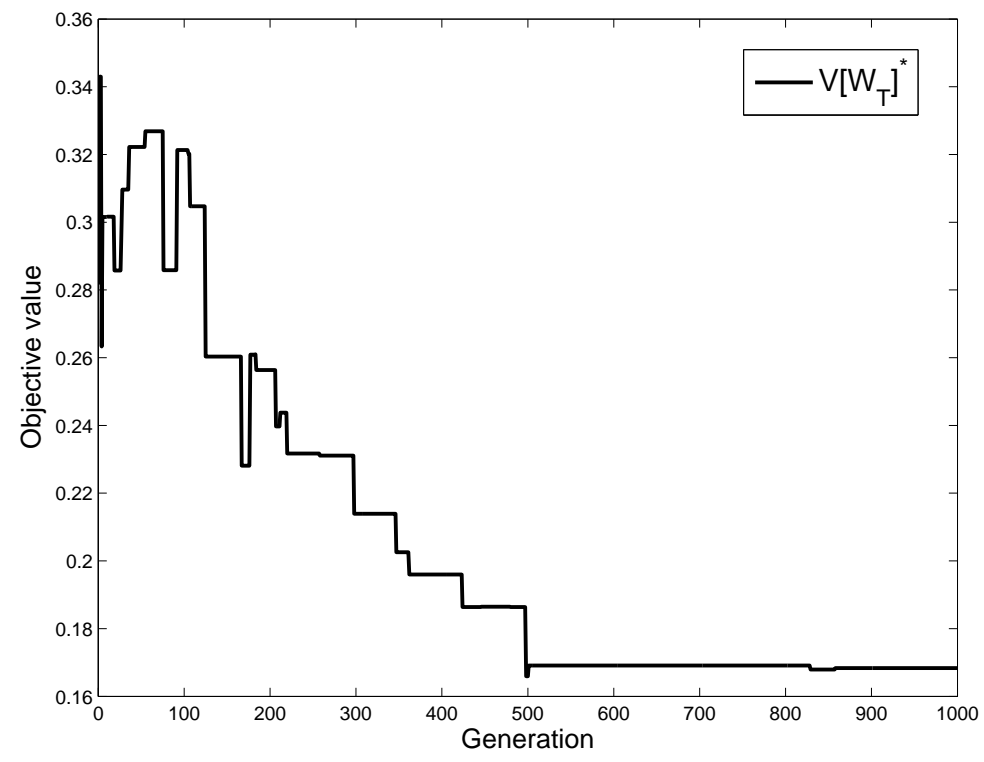

Figure 2: The convergence of genetic algorithm for optimal variance.

rates.

\section{Acknowledgments}

This work is supported by the National Natural Science Foundation of China (No.61673011).

\section{References}

[1] H. Markowitz, Portfolio selection, J. Financ. 7(1) (1952) 77-91.

[2] W.F. Sharpe, A linear programming algorithm for mutual fund portfolio selection, Manag. Sci. 13(7) (1967) 499-510.

[3] J.S. Pang, A new and efficient algorithm for a class of portfolio selection problems, Oper. Res. 28(3-part-ii) (1980) 754-767.

[4] M.J. Best, R.R. Grauer, Sensitivity analysis for mean-variance portfolio problems, Manag. Sci. 37(8) (1991) 980-989.

[5] M.R. Simonelli, Indeterminacy in portfolio selection, Eur. J. Oper. Res. 163(1) (2005) 170-176.

[6] B. Sawik, A three stage lexicographic approach for multi-criteria portfolio optimization by mixed integer programming, Prz. Elektrotechniczn. 84(9) (2008) 108-112. 
Table 5: The maximum final wealth, minimum risk of investment and optimal investment proportions for different weighting $\lambda$

\begin{tabular}{|c|c|c|c|}
\hline$\lambda$ & $\begin{array}{l}\text { Maximum final } \\
\text { wealth }\end{array}$ & $\begin{array}{l}\text { Minimum risk } \\
\text { of investment }\end{array}$ & $\begin{array}{c}\text { Optimal investment } \\
\text { proportions }\end{array}$ \\
\hline $10^{10}$ & 1.2719 & 0.1171 & $\begin{array}{c}\boldsymbol{x}_{1}^{*}=(0.0566,0.1815,0.0855,0.2913, \\
0.0290,0.1128,0.0243,0.2190) \\
\boldsymbol{x}_{2}^{*}=(0.0816,0.1559,0.0233,0.3043, \\
0.0010,0.0227,0.0262,0.3850) \\
\boldsymbol{x}_{3}^{*}=(0.1046,0.1548,0.2058,0.0026, \\
0.0452,0.0133,0.0984,0.3753) \\
\boldsymbol{x}_{4}^{*}=(0.2151,0.0915,0.0683,0.1547, \\
0.0826,0.0268,0.0033,0.3577)\end{array}$ \\
\hline $10^{8}$ & 1.2636 & 0.1331 & $\begin{array}{c}\boldsymbol{x}_{1}^{*}=(0.1848,0.0608,0.2251,0.0330, \\
0.0099,0.0425,0.0944,0.3495) \\
\boldsymbol{x}_{2}^{*}=(0.0407,0.0419,0.3037,0.0784, \\
0.0341,0.0986,0.0142,0.3884) \\
\boldsymbol{x}_{3}^{*}=(0.0819,0.2228,0.0252,0.1300, \\
0.0756,0.0129,0.0534,0.3982) \\
\boldsymbol{x}_{4}^{*}=(0.1052,0.0463,0.0741,0.0963, \\
0.0778,0.0904,0.1652,0.3447)\end{array}$ \\
\hline $10^{7}$ & 1.2602 & 0.1329 & $\begin{array}{c}\boldsymbol{x}_{1}^{*}=(0.0067,0.1164,0.2455,0.0725, \\
0.0661,0.0562,0.0455,0.3911) \\
\boldsymbol{x}_{2}^{*}=(0.0249,0.0866,0.2651,0.0232, \\
0.1979,0.0033,0.0128,0.3862) \\
\boldsymbol{x}_{3}^{*}=(0.2035,0.0091,0.3033,0.0569, \\
0.0559,0.0144,0.0527,0.3042) \\
\boldsymbol{x}_{4}^{*}=(0.0736,0.0364,0.0328,0.0546, \\
0.2257,0.1340,0.0442,0.3987)\end{array}$ \\
\hline $10^{6}$ & 1.2574 & 0.1427 & $\begin{array}{c}\boldsymbol{x}_{1}^{*}=(0.1479,0.1548,0.0295,0.0444, \\
0.0948,0.1411,0.0169,0.3706) \\
\boldsymbol{x}_{2}^{*}=(0.2507,0.0397,0.0970,0.0023, \\
0.0361,0.1966,0.0589,0.3187) \\
\boldsymbol{x}_{3}^{*}=(0.3035,0.0066,0.0587,0.0705, \\
0.1532,0.0083,0.0118,0.3874) \\
\boldsymbol{x}_{4}^{*}=(0.1554,0.0329,0.0158,0.1222, \\
0.0315,0.2727,0.0762,0.2933)\end{array}$ \\
\hline $10^{5}$ & 1.2507 & 0.1404 & $\begin{array}{c}\boldsymbol{x}_{1}^{*}=(0.1327,0.0039,0.2539,0.0959, \\
0.0172,0.0071,0.1011,0.3882) \\
\boldsymbol{x}_{2}^{*}=(0.0018,0.1907,0.3076,0.0190, \\
0.0537,0.0137,0.0750,0.3385) \\
\boldsymbol{x}_{3}^{*}=(0.1259,0.0135,0.0984,0.3680, \\
0.0280,0.0197,0.0388,0.3077) \\
\boldsymbol{x}_{4}^{*}=(0.1139,0.0197,0.0226,0.1237, \\
0.1280,0.0227,0.1799,0.3895)\end{array}$ \\
\hline
\end{tabular}


[7] B. Sawik, Selected multi-objective methods for multiperiod portfolio optimization by mixed integer programming, in: K.D. Lawrence, G. Kleinman (Eds.), Applications of Management Science, Emerald Group Publishing Ltd., Bradford, 2010, pp. 3-34.

[8] B. Sawik, Bi-criteria portfolio optimization models with percentile and symmetric risk measures by mathematical programming, Prz. Elektrotechniczn. 88(10B) (2012) 176-180.

[9] Y.F. Sun, G. Aw, K.L. Teo, G.L. Zhou, Portfolio optimization using a new probabilistic risk measure, J. Ind. Manag. Optim. 11(4) (2015) 1275-1283.

[10] D. Li, W.L. Ng, Optimal dynamic portfolio selection: multiperiod mean-variance formulation, Math. Financ. 10(3) (2000) 387-406.

[11] S.S. Zhu, D. Li, S.Y. Wang, Risk control over bankruptcy in dynamic portfolio selection: a generalized mean-variance formulation, IEEE Trans. Autom. Control 49(3) (2004) 447-457.

[12] G.C. Calafiore, Multi-period portfolio optimization with linear control policies, Automatica 44(10) (2008) 2463-2473.

[13] O.L.V. Costa, M.V. Araujo, A generalized multi-period mean-variance portfolio optimization with Markov switching parameters, Automatica 44(10) (2008) 2487-2497.

[14] H.L. Wu, Z.F. Li, Multi-period mean-variance portfolio selection with Markov regime switching and uncertain time-horizon, J. Syst. Sci. Complex. 24(1) (2011) 140-155.

[15] H.L. Wu, Z.F. Li, Multi-period mean-variance portfolio selection with regime switching and a stochastic cash flow, Insur. Math. Econ. 50(3) (2012) 371-384.

[16] Y.F. Sun, G. Aw, K.L. Teo, Y.J. Zhu, X.Y. Wang, Multi-period portfolio optimization under probabilistic risk measure, Financ Res. Lett. 18 (2016) 60-66.

[17] E. Vercher, J.D. Bermdez, J.V. Segura, Fuzzy portfolio optimization under downside risk measures, Fuzzy Sets Syst. 158(7) (2007) 769-782.

[18] X. Li, Z.F. Qin, S. Kar, Mean-variance-skewness model for portfolio selection with fuzzy returns, Eur. J. Oper. Res. 202(1) (2010) 239-247.

[19] S.J. Sadjadi, S.M. Seyedhosseini, K. Hassanlou, Fuzzy multi period portfolio selection with different rates for borrowing and lending, Appl. Soft Comput. 11(4) (2011) 3821-3826.

[20] Y.J. Liu, W.G. Zhang, W.J. Xu, Fuzzy multi-period portfolio selection optimization models using multiple criteria, Automatica 48(12) (2012) 3042-3053.

[21] W.G. Zhang, Y.J. Liu, W.J. Xu, A possibilistic mean-semivariance-entropy model for multiperiod portfolio selection with transaction costs, Eur. J. Oper. Res. 222(2) (2012) 341-349.

[22] Y.J. Liu, W.G. Zhang, A multi-period fuzzy portfolio optimization model with minimum transaction lots, Eur. J. Oper. Res. 242(3) (2015) 933-941.

[23] S. Guo, L. Yu, X. Li, S. Kar, Fuzzy multi-period portfolio selection with different investment horizons, Eur. J. Oper. Res. , 254(3) (2016) 1026-1035. 
[24] M.K. Mehlawat, Credibilistic mean-entropy models for multi-period portfolio selection with multi-choice aspiration levels, Inf. Sci. 345 (2016) 9-26.

[25] X. Huang, H. Ying. Risk index based models for portfolio adjusting problem with returns subject to experts' evaluations, Econ. Model. 30(30) (2013) 61-66.

[26] B.D. Liu, Uncertainty Theory, Second ed., Springer-Verlag, Berlin, 2007.

[27] B.D. Liu, Uncertainty Theory: A Branch of Mathematics for Modeling Human Uncertainty, Springer-Verlag, Berlin, 2010.

[28] B.D. Liu, Some research problems in uncertainty theory, J. Uncertain Syst. 3 (2009) 3-10.

[29] K. Yao, X. Li, Uncertain alternating renewal process and its application, IEEE Trans. Fuzzy Syst. 20(6) (2012) 1154-1160.

[30] Y. Gao, L. Yang, S. Li, Uncertain models on railway transportation planning problem, Appl. Math. Model. 40(7-8) (2015) 4921-4934.

[31] H.Y. Yan, Y.G. Zhu, Bang-bang control model for uncertain switched systems, Appl. Math. Model. 39(10-11) (2015) 2994-3002.

[32] K. Yao, A formula to calculate the variance of uncertain variable, Soft Comput. 19(10) (2015) 2947-2953.

[33] Z.F. Qin, S. Kar, H. Zheng, Uncertain portfolio adjusting model using semiabsolute deviation, Soft Comput. 20(2) (2016) 717-725.

[34] Y.G. Zhu, Uncertain optimal control with application to a portfolio selection model, Cybern. Syst. 41(7) (2010) 535-547.

[35] Y.F. Ning, L.M. Yan, Y.H. Xie, Mean-TVaR model for portfolio selection with uncertain returns, Information 15 (2012) 129-137.

[36] K. Yao, X.Y. Ji, Uncertain decision making and its application to portfolio selection problem, Int. J. Uncertain Fuzziness Knowl. Based Syst. 22(1) (2014) 113-123.

[37] Y.F. Ning, H. Ke, Z. Fu, Triangular entropy of uncertain variables with application to portfolio selection, Soft Comput. 19(8) (2015) 2203-2209.

[38] X. Huang, L. Qiao, A risk index model for multi-period uncertain portfolio selection, Inf. Sci. $217(24)(2012) 108-116$.

[39] J.H. Holland, Adaptation in Natural and Artificial Systems, University of Michigan Press, New York, 1975.

[40] X. Huang, Two new models for portfolio selection with stochastic returns taking fuzzy information, Eur. J. Oper. Res. 180(1) (2007) 396-405.

[41] W. Yan, R. Miao, S.R. Li, Multi-period semi-variance portfolio selection: model and numerical solution, Appl. Math. Comput. 194(1) (2007) 128-134. 
[42] B. Wang, S.M. Wang, J. Watada . Fuzzy-portfolio-selection models with value-at-risk, IEEE Trans. Fuzzy Syst, 19(4) (2011) 758-769.

[43] W.G. Zhang,Y.J. Liu, Credibilitic mean-variance model for multi-period portfolio selection problem with risk control, OR Spectr. 36(1) (2014) 113-132. 